\title{
dspace.vutbr.cz
}

\section{Adjacent Channel Interference Cancellation in FDM Transmissions}

HARVÁNEK, M.; MARŠÁLEK, R.; KRÁL, J.; GÖTTHANS, T.; BLUMENSTEIN, J.; POSPÍŠIL, M.; RUPP, M.

IEEE Transactions on Circuits and Systems I: Regular Papers

2020 , vol. 67 , iss. 12 pp. $5417-5428$

ISSN: 1558-0806

DOI: http://dx.doi.org/10.1109/TCSI.2020.2995350

Accepted manuscript

(C) 2020 IEEE. Personal use of this material is permitted. Permission from IEEE must be obtained for all other uses, in any current or future media, including reprinting/republishing this material for advertising or promotional purposes, creating new collective works, for resale or redistribution to servers or lists, or reuse of any copyrighted component of this work in other works. 


\title{
Adjacent Channel Interference Cancellation in FDM Transmissions
}

\author{
Michal Harvanek ${ }^{\circledR}$, Roman Marsalek ${ }^{\circledR}$, Member, IEEE, Jan Kral ${ }^{\circledR}$, Tomas Gotthans ${ }^{\circledR}$, Member, IEEE, \\ Jiri Blumenstein ${ }^{\circledR}$, Member, IEEE, Martin Pospisil $^{\circledR}$, Markus Rupp ${ }^{\circledR}$, Fellow, IEEE
}

\begin{abstract}
Interference between users in adjacent channels negatively affects throughput of mobile networks. In this paper we aim at cancellation of interference caused by a nonlinear power amplifier in a generalized orthogonal frequency division system. We propose an interference cancellation method to subtract these out-of-band emissions from the received signal. In contrast to state-of-the-art methods, our proposed method employs over-the-air estimation of power amplifier model parameters together with a particular frequency domain filtering method that allows to generate the required training data. The proposed interference cancellation method is also verified by an experiment on a software defined radio test bench.
\end{abstract}

\section{Index Terms}

Generalized frequency division multiplex, interference cancellation, nonlinear power amplifier, channel estimation error, outof-band emission, over-the-air estimation.

\section{INTRODUCTION}

D UE to its immunity to multipath propagation, Orthogonal Frequency Division Multiplexing (OFDM) has been widely applied in the 4th generation of wireless mobile communication systems and in wireless local area networks.

In contrast to its advantages, such as a simple one-tap equalization on the subcarriers and easy implementation by the Inverse Fast Fourier Transform (IFFT), the OFDM technique also imposes some serious drawbacks. High Peak to Average Power Ratio (PAPR) reduces the transmitter power efficiency, the need for a Cyclic Prefix (CP) for every individual OFDM symbol limits the spectral efficiency and the significant Out-Of-Band $(\mathrm{OOB})$ radiations due to the $\sin x / x$ nature of the subcarrier signals prevent the application of OFDM in opportunistic scenarios with fragmented spectrum.

In the last decade, there has been intensive research of multicarrier waveforms with improved spectral properties, relaxing the strong complex orthogonality requirement of OFDM. One way to achieve better spectral properties is to follow a filter bank approach as in the Filter Bank Multi Carrier (FBMC), including Offset Quadrature Amplitude Modulation (OQAM) [1] with alternating real and imaginary symbols on the adjacent subcarriers. Filtering applied to blocks of neighbouring subcarriers has been proposed in [2] as an Universal Filtered Multi Carrier (UFMC), or later in a similar approach called Filtered OFDM (F-OFDM) [3], [4]. Another flexible alternative to OFDM has been proposed in [5] as Generalized Frequency Division Multiplexing (GFDM) that can cover both CP-OFDM and Single Carrier with Frequency Domain Equalization (SC-FDE) as its extreme cases. Unfortunately, the low $\mathrm{OOB}$ emission of above-mentioned waveforms deteriorates by the imperfections of a

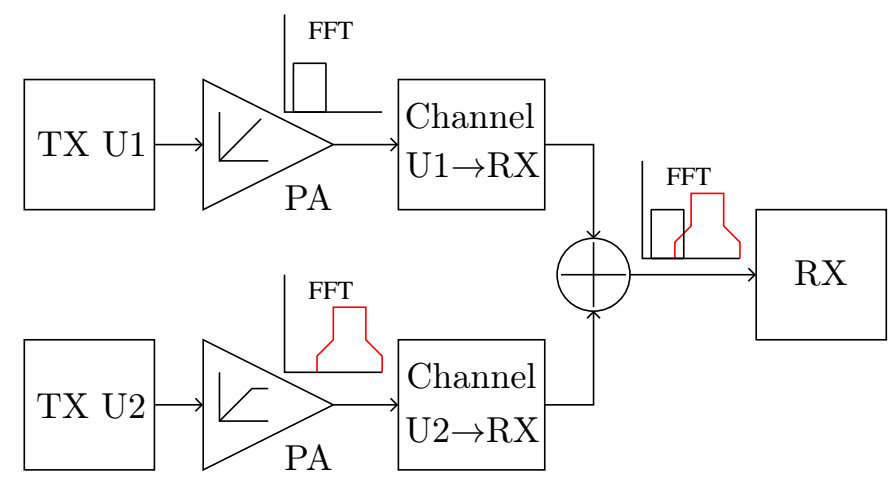

Fig. 1. Interference scenario.

radio frequency front-end, e.g., by the nonlinearity of a Power Amplifier (PA) [6]. The nonlinear distortion of OFDM signals

Manuscript received ...; revised ... . The research presented in this paper was financed by the Czech Science Foundation (GACR) project 17-18675S Future transceiver techniques for the society in motion and partially supported by the Czech Ministry of Education within the frame of the National Sustainability Program grant LO1401 (INWITE) and by a Brno University of Technology internal project FEKT-S-20-6325

All the authors are with the Department of Radio Electronics, Brno University of Technology, Brno, Czech Republic (e-mails: \{xharva02,xpospi29\}@stud.feec.vutbr.cz, \{marsaler,gotthans,blumenstein\}@feec.vutbr.cz, jan.kral@vutbr.cz). Markus Rupp is also with Institute of Telecommunications, Technische Universität Wien, Vienna, Austria. 
caused by the power amplifier has been thoroughly studied in the past [7], [8], [9] and various approaches have been proposed to eliminate it including a digital pre-distortion [10] at the transmitter side. At the receiver side, Interference Cancellation (IC) [11] methods based on signal detection and estimation of in-band nonlinear distortion components or maximum likelihood detection [12] methods have been developed for OFDM systems to eliminate the effect of nonlinear distortions.

Recently, research on IC methods has also focused towards the application to multicarrier modulation waveforms. A GFDM receiver with single-user nonlinear in-band distortion cancellation has been studied in [13]. A method based on the Bussgang's theorem model of the PA output as the complex gain and the additive Gaussian noise component was presented in [14], with the widely-used assumption on perfect knowledge of the PA model.

Besides nonlinear distortion cancellation, IC has also been an alternative to FBMC channel equalization [15]. A similar approach has also been proposed for Self-Interference Cancellation (SIC) between non-orthogonal subcarriers [16] or for iterative frequency domain equalization [17].

In many methods presented above, the estimation of the PA model at the receiver is necessary. In a real fading channel, this is a challenging task and very few attempts to adapt the PA model over-the-air have been reported so far [18], [19].

With the advent of communications between machines, particularly Vehicle-to-Everything (V2X) communications, the number of connected wireless devices is rapidly growing and the problem of Adjacent Channel Interference (ACI) becomes accentuated, see e.g. a recent report on 5G V2X Radio [20]. Various multicarrier waveforms have been compared with respect to their robustness against the interference from neighbouring users [21], but the effective mitigation of ACI effects is still an open research issue.

The general idea of subtracting the gain-compensated interference signal from the useful signal has been proposed in [22] with no details about how the replica of the interference signal is generated. The adjacent channel interference suppression by a selective application of pre-calculated filters has been published in patent application [23], with the adjacent channel interference detection by a simple energy detector. In [24], the adaptive ACI estimation based on comparing the spectrum of the received signal with the spectrum of known pilot signals has been proposed, thus reducing the overall throughput. The authors of [25] considered a dual satellite communication system with a Volterra filter-based cancellation of first and third order interference terms for narrowband single-carrier signals. Similarly to our approach, the generation of the interference signal replica is considered in the ACI cancellation method proposed in [26] for single-carrier signals. The memoryless characteristic of the interfering transmitter PA was estimated from the received data signals, as the pilot signals usually have much lower PAPR. In contrast to our approach, the separation of single carrier signals of both received users is done prior the downconversion to the baseband by a pair of analog band-pass filters. A multi-user interference cancellation is used for the compensation of interferences caused by the carrier frequency offset in OFDMA uplink receivers [27], but this approach does not include the cancellation of interferences caused by the nonlinear PA.

The main contributions of this paper are as follows:

- we propose a novel IC method based on the subtraction of nonlinearity-induced interference from adjacent users and over-the-air estimation of the PA model with digital frequency domain filtering and we tailor it to FDM systems.

- we evaluate the proposed method by extensive simulation according to the scenario depicted by Fig. 1 with various cases of linearly or nonlinearly amplified users and varying power difference of the users. We also investigate the influence of channel estimation error and effect of multipath propagation parameters on the performance of the method.

- we experimentally verify the method using a Software Defined Radio (SDR) platform.

\section{Adjacent Channel Interference SCEnARio}

Similarly to a recent study on adjacent channel interference in 5G V2X communications [20, p. 44], we adopt the scenario of two co-located users adjacent to each other in the frequency domain as depicted in Fig. 1. In the following, a link between transmitter $\mathrm{U} 1$ and receiver $\mathrm{RX}(\mathrm{U} 1 \rightarrow \mathrm{RX})$ is the main link of interest. In addition, we suppose the existence of a second transmitter U2, in the frequency domain adjacent to the main link, see Fig. 2. Due to the nonlinear power amplifier of U2, this transmitter acts as an interferer to the main link. The increased OOB emissions from U2, characterized by the degraded Adjacent Channel Protection Ratio (ACPR), result in a degraded performance of the main link. Note the ACPR is usually defined [28] as a ratio between the integrated power in the adjacent channel to the integrated power in the main channel expressed in logarithmic units:

$$
\text { ACPR }[\mathrm{dB}]=10 \cdot \log _{10} \frac{\int P_{\text {adjacent }} \mathrm{d} f}{\int P_{\text {main }} \mathrm{d} f} .
$$

Hereinafter we consider an equal bandwidth of the main and adjacent channels and separation between the channels of $\Delta f$. This degradation manifests in a poor Bit Error Ratio (BER) and can also be quantified as an increased Error Vector Magnitude $(E V M)$. The degradation of the EVM performance by the interference of U2 depends on the Guard Band (GB) which defines spacing between users in terms of the number of subcarriers, the ACPR of U2 and the power difference $\Delta P$ between the two transmitters defined as:

$$
\Delta P[\mathrm{~dB}]=P_{\mathrm{U} 2}[\mathrm{dBm}]-P_{\mathrm{U} 1}[\mathrm{dBm}] .
$$

The variable $K_{\text {data }}$ denotes the number of data subcarriers for each user. These terms are illustrated in Fig. 2. 


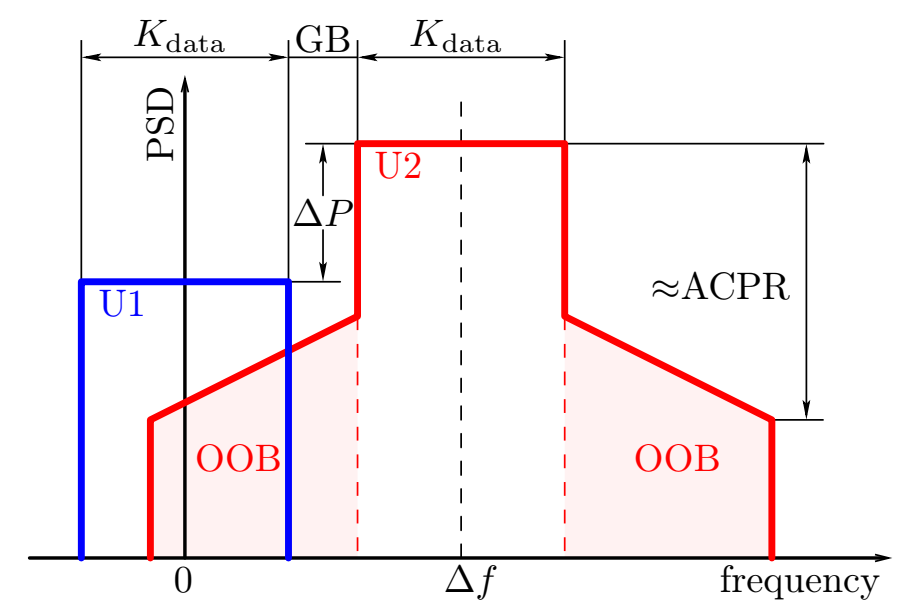

Fig. 2. Definition of scenario parameters - GB, ACPR, power difference $\Delta P$ between users U1 and $\mathrm{U} 2$, frequency spacing between users $\Delta f$.

\section{Proposed Interference CANCELler}

Similarly to state-of-the-art IC methods [13], [14], the proposed approach is based on the detection of nonlinearly distorted data, their re-modulation and subtraction of the distortion from the received signal. The source of interference expected hereinafter is not the PA of U1, but the PA in the adjacent transmitter (U2) causing the interference to RX from outside the band. In addition, the proposed method includes the estimation of the interfering PA modeled by a memory polynomial model [29]. In contrast to the state-of-the-art [26], the proposed canceller does not use band separation in the analog domain negatively affected by the imprecise band-pass filters, but rather a tailored frequency-domain digital filter. In the following, a matrix form of modulation [15], [30]:

$$
\mathbf{y}_{u}=\mathbf{A d}_{u},
$$

and demodulation

$$
\mathbf{d}_{u}=\mathbf{B} \mathbf{y}_{u}
$$

is used, with $u$-th user $L \times 1$ data vector $\mathbf{d}_{u}=\left[\mathbf{d}_{u, 0} \ldots \mathbf{d}_{u, L-1}\right]^{\mathrm{T}}$, corresponding $L \times 1$ time domain samples vector $\mathbf{y}_{u}=$ $\left[\mathbf{y}_{u, 0} \ldots \mathbf{y}_{u, L-1}\right]^{\mathrm{T}}$ and $L \times L$ modulation and demodulation matrices $\mathbf{A}$ and $\mathbf{B}$. Relations of vector and matrix dimensions to the system parameters (number of symbols and subcarriers) are modulation-dependent and are specified in detail in the Section IV.

Although the receiver's RX interest is to demodulate the data of user U1, it needs to simultaneously receive both transmitted signals and process the user U2 signal to suppress the interference caused by U2. The complex baseband signal received at $\mathrm{RX}$ is:

$$
\mathbf{y}_{\mathbf{r}}=G \mathbf{y}_{\mathbf{1}}+\mathcal{A}\left\{\mathbf{y}_{2}\right\}^{\Delta f}+\boldsymbol{\eta} .
$$

It consists of a linearly amplified (with a complex scalar gain $G$ ) user U1 signal $\mathbf{y}_{1}$ transmitted at zero frequency and a nonlinearly amplified (with an amplifier $\mathcal{A}$, see subsection III-A) interferer $\mathcal{A}\left\{\mathbf{y}_{2}\right\}^{\Delta f}$ whose frequency is shifted by $\Delta f$ from the zero frequency of $\mathbf{y}_{\mathbf{2}}$. The symbol $\boldsymbol{\eta}$ denotes an $L \times 1$ Additive White Gaussian Noise (AWGN) vector. The method is

also applicable to the case of both U2 and U1 being amplified with the nonlinear power amplifiers, as we demonstrate on BER evaluations in Section VI-B.

\section{A. Core of the IC algorithm}

The goal of the IC method is to suppress the OOB emissions of U2 into the band of U1. The block scheme of the IC system is depicted in Fig. 3.

The core of the algorithm works as follows:

\section{Interfering user data detection}

The received signal $\mathbf{y}_{r}$ is frequency shifted by $-\Delta f$, denoted as $\mathbf{y}_{r}^{-\Delta f}$ and demodulated according to (4). In vector form, we can write:

$$
\hat{\mathbf{d}}_{2}=\mathbf{B y}_{r}^{-\Delta f},
$$

where $\hat{\mathbf{d}_{2}}$ is a detected vector of $\mathrm{U} 2$ data. Both $\hat{\mathbf{d}}_{2}$ and $\mathbf{y}_{r}^{-\Delta f}$ are $L \times 1$ vectors.

\section{Interfering user data re-modulation}




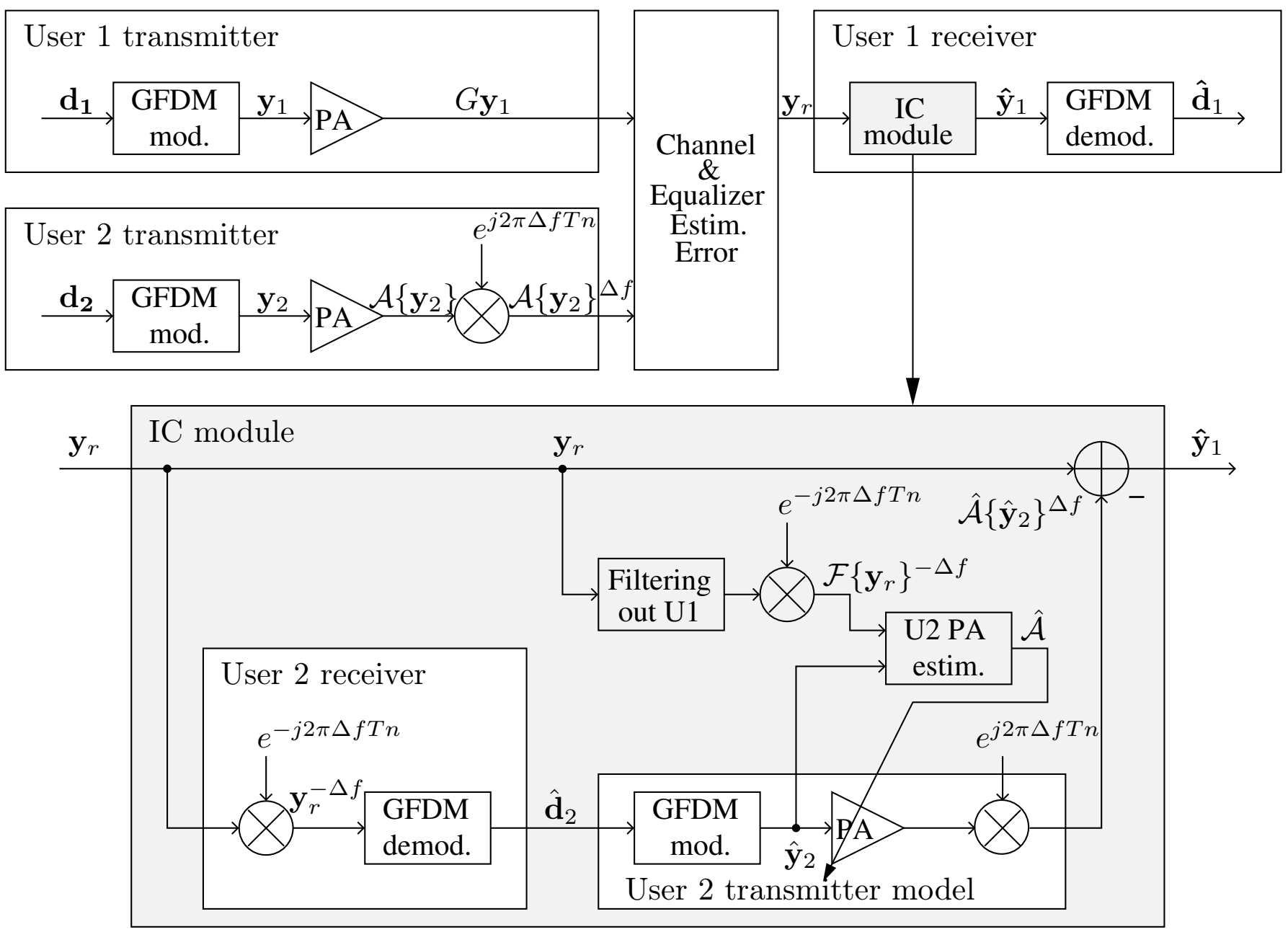

Fig. 3. Baseband model block diagram depicting two interfering users and our proposed method for reducing the interference of U2 to U1. $T$ denotes sampling period.

Estimate $\hat{\mathbf{d}}_{2}$ is re-modulated according to (3):

$$
\hat{\mathbf{y}}_{2}=\mathbf{A} \hat{\mathbf{d}}_{2} .
$$

3. Nonlinear PA estimation For a low complexity, but yet satisfactory performance, we have considered a memory polynomial baseband model of U2 PA [29] :

$$
\hat{\mathcal{A}}\left\{\hat{y}_{2}[n]\right\}=\sum_{p=1}^{P} \sum_{q=0}^{Q} \hat{b}_{p, q} \hat{y}_{2}[n-q]\left|\hat{y}_{2}[n-q]\right|^{p-1}
$$

with $P$ describing the polynomial order, $Q$ the memory depth and $\hat{b}_{p, q}$ being the PA model coefficients estimates.

The parameters of $\hat{\mathcal{A}}$ are estimated by the standard least squares method [29] requiring knowledge of the PA input estimate $\hat{\mathbf{y}}_{2}$ and the PA output. The output signal $\mathbf{y}_{r, \mathcal{F}}$ of the U2 PA is obtained by filtering-out the signal $G \mathbf{y}_{1}$ from the received signal $\mathbf{y}_{r}$ by the filter $\mathcal{F}\{\}$ :

$$
\mathbf{y}_{r, \mathcal{F}}=\mathcal{F}\left\{\mathbf{y}_{r}\right\} .
$$

Note that $\mathbf{y}_{r, \mathcal{F}}$ is a bandpass signal centered at $\Delta f$ and needs to be shifted to baseband by $-\Delta f$ for the adaptation of the baseband PA model. For more details on the proposed filtering approach, see Section III-B.

4. Interference subtraction

Based on the estimated baseband signal $\hat{\mathbf{y}}_{2}$ and the PA model $\hat{\mathcal{A}}$, the interference signal $\hat{\mathcal{A}}\left\{\hat{\mathbf{y}}_{2}\right\}^{\Delta f}$ is generated and subtracted from the received signal $\mathbf{y}_{r}$ :

$$
\hat{\mathbf{y}}_{1} \approx \mathbf{y}_{r}-\hat{\mathcal{A}}\left\{\hat{\mathbf{y}}_{2}\right\}^{\Delta f} .
$$

\section{User 1 data detection}




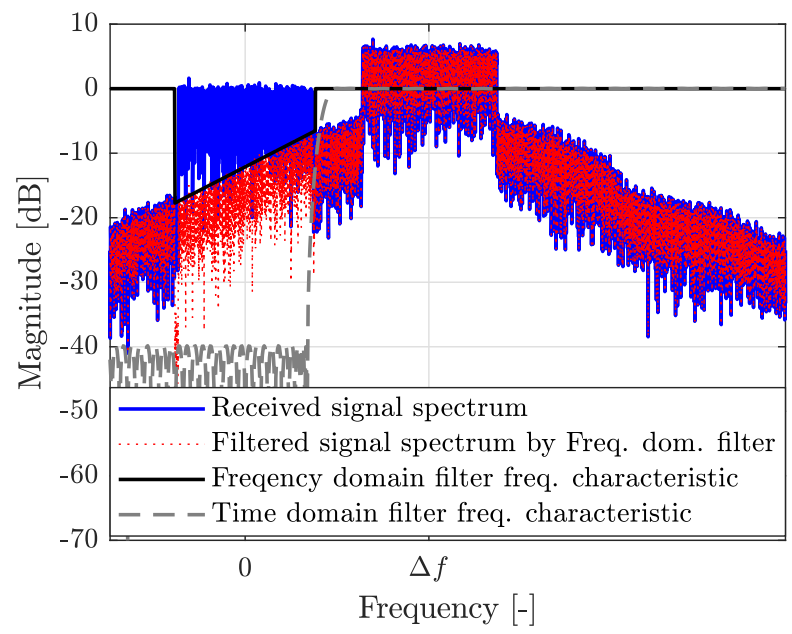

Fig. 4. Frequency characteristics of implemented filters.

As the final step, data $\hat{\mathbf{d}}_{1}$ of user $\mathrm{U} 1$ are detected according to Eq. (4)

$$
\hat{\mathbf{d}}_{1}=\mathbf{B} \hat{\mathbf{y}}_{1} \text {. }
$$

\section{B. Time Domain and Frequency Domain Filtering}

The signal of user $\mathrm{U} 1$ is filtered out from the composite received signal $\mathbf{y}_{\mathbf{r}}$ by a Finite Impulse Response (FIR) filter for the task of U2 power amplifier estimation. To this end we have developed and tested filters in both the time and frequency domain.

1. Time Domain Filtering To filter a signal in the time domain, a FIR filter was applied. The filter output can be expressed in matrix form as

$$
\mathcal{F}\left\{\mathbf{y}_{r}\right\}=\mathbf{F} \cdot \mathbf{y}_{r}
$$

where $\mathbf{F}$ is a Toeplitz matrix with its first column composed of the filter coefficients and $\mathbf{y}_{r}$ is a received time domain signal vector. An example of the frequency response of such an FIR filter is shown in Fig. 4 by the gray dashed line.

2. Frequency Domain Filtering

Due to spectral regrowth by nonlinearity, the PA model estimation needs to be performed on the signals captured with at least three times greater bandwidth than the original [31]. The time domain filtering by the FIR filter is thus not a good option as it cancels the intermodulation products outside the U2 main channel. Nevertheless, the signal of U1 still needs to be filtered out.

We have thus proposed and evaluated the frequency domain filter w with a variable frequency characteristic. The frequency domain filtering is defined by:

$$
\mathcal{F}\left\{\mathbf{y}_{r}\right\}=\operatorname{IFFT}\left\{\mathbf{w} \cdot \operatorname{FFT}\left\{\mathbf{y}_{r}\right\}\right\}
$$

where (I)FFT denotes the (Inverse) Fast Fourier Transform pair and w is a vector of frequency domain filter coefficients. The filter frequency characteristic is dependent on the power difference $\Delta P$ between users and the slope of the spectrum of the $\mathrm{U} 2$ adjacent channel. The goal is to filter out $\mathrm{U} 1$ from the composite signal $y_{r}$ while preserving the $\mathrm{U} 2$ spectrum symmetry as is shown in Fig. 4. The blue solid curve depicts the spectrum of the received composite signal. The spectrum of the filtered signal is depicted by a red dotted curve. The filter frequency characteristic is depicted by a black line. To simplify the filter design, we assume a flat main channel of U1 and a constant slope of U2 spectrum in its adjacent channel.

\section{GFDM MODULATION AND DEMODULATION}

In the case of GFDM on which we demonstrate the proposed IC method, $\mathbf{d}_{u}$ is the vector of data symbols $d_{m, k}$ transmitted on the $k$-th subcarrier and the $m$-th subsymbol, [5]. The modulation matrix $\mathbf{A}$ is a $[K M \times K M]$ matrix, where $K$ is the total number of subcarriers and $M$ is the total number of subsymbols. This matrix consists of frequency and time shifted versions of a prototype filter, i.e., $\mathbf{A}=\left[\mathbf{g}_{0,0} \ldots \mathbf{g}_{K-1,0} \ldots \mathbf{g}_{K-1, M-1}\right]$. The elements of the vectors $\mathbf{g}_{k, m}=\left(g_{k, m}[n]\right)^{T}, n=0, \ldots, L-1$ defining the prototype filter can be written as:

$$
g_{k, m}[n]=g([n-m K] \bmod L) \cdot w^{-k n},
$$




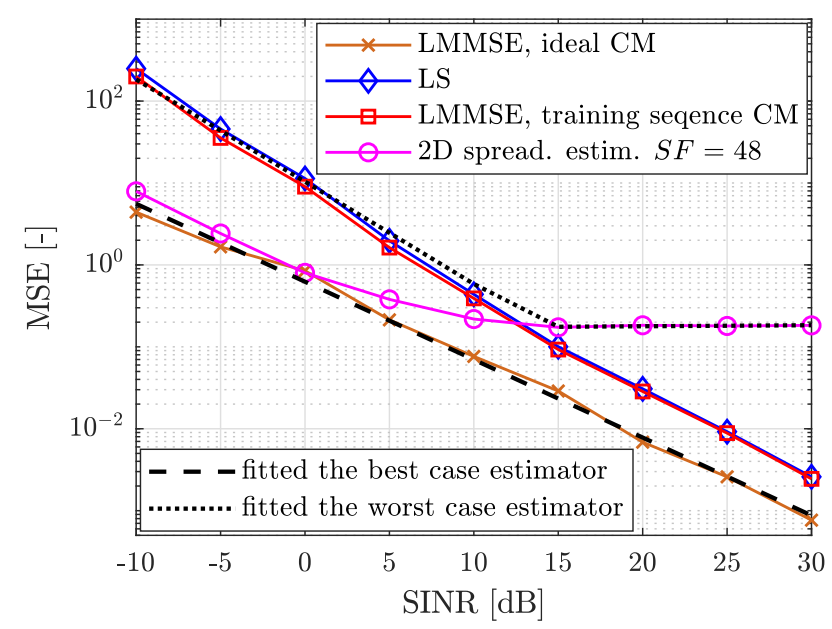

Fig. 5. MSE of selected channel estimators (in color), adopted from [32] together with fitted LMMSE with ideal CM as the best case estimator and hypothetically the worst case estimator as a combination of the LS estimator and 2D spreading estimator.

where $L=K M$ is the number of symbols and $w^{k n}=e^{j 2 \pi \frac{k}{K} n}$ describes the frequency shift for the subcarrier.

There are two basic options how to design a demodulation matrix $\mathbf{B}$ [5]. The first one is a zero-forcing receiver, i.e., $\mathbf{B}=\mathbf{A}^{-1}$. The second common option is to employ a matched filter demodulator, with $\mathbf{B}=\mathbf{A}^{\mathrm{H}}$, where $(.)^{\mathrm{H}}$ is the Hermite transpose operator. To suppress the effect of inband self-interference the zero-forcing receiver is employed in simulation and experiment.

\section{Model of the Equalizer ERror}

The over-the-air nonlinear PA estimation feasibility has been recently experimentally demonstrated in the application of digital pre-distortion of phased array transmitters [19], however with only a rude analysis about the influence of the fading channel on the over-the-air performance.

Equalization is necessary in a real channel, accurate Channel State Information (CSI) is typically not available. Thus, we analyzed the performance of the proposed method in the presence of imperfect CSI. We model a channel-equalizer chain with imperfect CSI by the Mean Square Error (MSE) of the channel estimate defined as [33]

$$
\operatorname{MSE}=E\left[\left|\mathbf{h}_{u}-\hat{\mathbf{h}}_{u}\right|^{2}\right],
$$

where $\mathbf{h}_{u}$ and $\hat{\mathbf{h}}_{u}$ are the true and estimated frequency domain coefficients of the channel between the receiver RX and $u$-th user transmitter.

The numerical value of MSE depends on the channel estimator and Signal to Noise Ratio (SNR). For several typical estimators, we have adopted the MSE curves as a function of SNR [32], see Fig. 5. The brown and red curves depict the Linear Minimum Mean Square Error (LMMSE) estimator based on an ideal Covariance Matrix (CM) and estimator based on two-dimensional spreading with Spreading Factor $(\mathrm{SF})=48$, respectively. The blue curve depicts a Least Squares (LS) estimator error and the red curve describes the LMMSE with CM calculated from a training sequence. We have considered the channel estimation error due to additive noise, but also due to the additional interference by the user U2, depending on the U2 PA working point. In the following, MSE thus depends on the Signal to Interference and Noise Ratio (SINR).

Assuming the wireless channel being frequency flat over GFDM subcarriers, we model the effect of an imperfect channelequalizer chain on the time domain vector $\mathbf{y}_{u}$ as:

$$
\mathbf{y}_{u, \text { imperfect }}=\operatorname{IFFT}\left\{\Omega \cdot \operatorname{FFT}\left\{\mathbf{y}_{u}\right\}\right\},
$$

with $\Omega$ denoting an $L \times 1$ vector of independent, identically distributed (i.i.d.) complex Gaussian entries with unit mean and variance equal to channel estimate MSE.

\section{Vi. Evaluation by Simulation}

Both GFDM modulators [34] of U1 and U2 apply the same settings. One GFDM symbol contains $K=4096$ subcarriers, $K_{\text {data }}=204$ out of 4096 subcarriers contain data symbols to resemble 17 LTE downlink resource blocks [35]. The remaining subcarriers are carrying zero symbols, introduced to accommodate the frequency space for the second user and for OOB radiation due to the PA. Zero symbols are symmetrically placed on both lower and upper edges of the spectrum. The total 


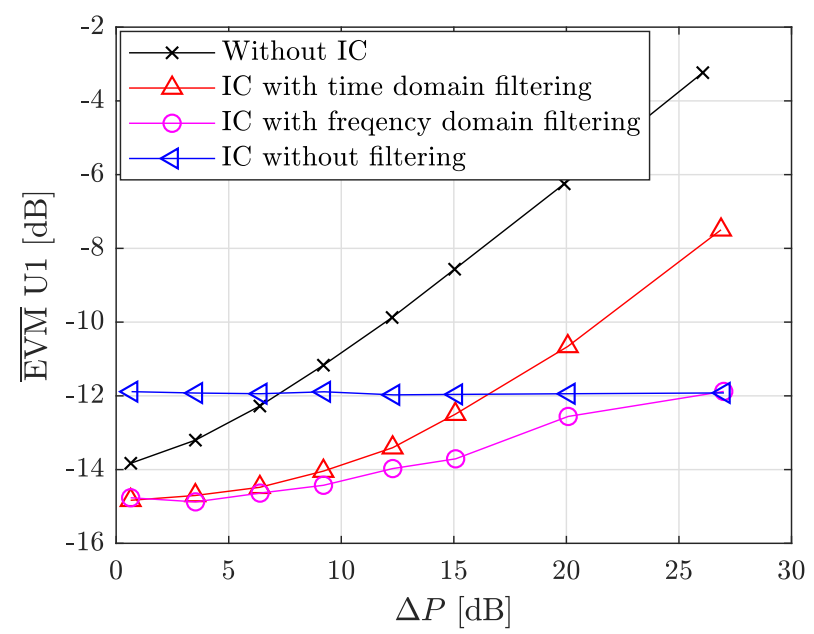

Fig. 6. IC method performance for AWGN channel, user U1 SNR=30 dB.

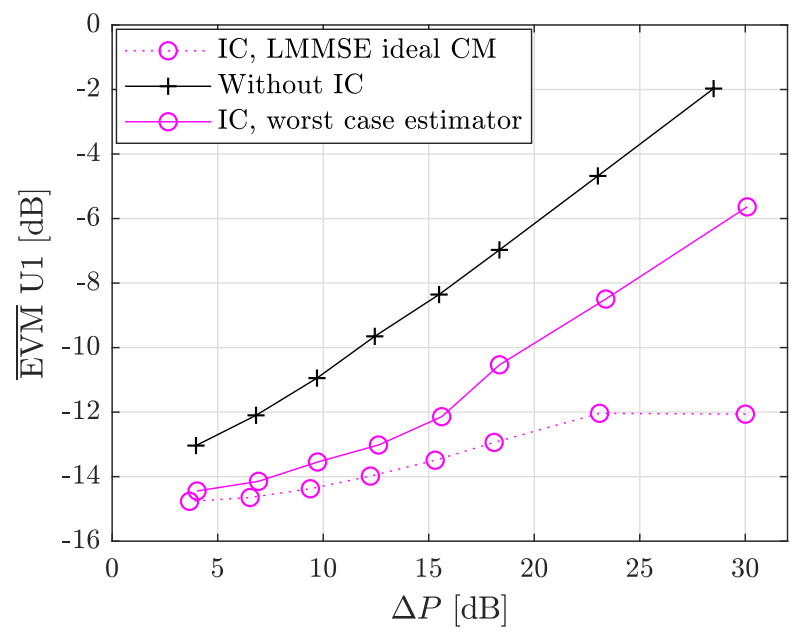

Fig. 7. IC method performance employing frequency filtering, channel estimation error applied to U2, perfect CSI knowledge of U1 for the best and worst case channel estimators, user U1 SNR=30 dB.

number of subsymbols is $M=15$. The individual subcarriers are mapped according to the QPSK (Sections VI-A and VII) or 16-QAM (Section VI-B) constellation diagram. The Root Raised Cosine (RRC) filter with a roll off factor of 0.3 defines filter $g$.

The PAPR of the tranmitted signals is approximately $12 \mathrm{~dB}$. The frequency shift $\Delta f$ of $\mathrm{U} 2$ is chosen such as a guard band between the users corresponds to a 75 subcarrier width. The nonlinear user (U2) ACPR is $38 \mathrm{~dB}$.

A memory polynomial model according to Eq. (8) with $P=7$ and $Q=1$ for nonlinear PA ADL5610 has been extracted from the measurements on an NI USRP 2944R SDR with UBX front-end set to a sampling frequency of $20 \mathrm{MHz}$. The carrier frequency was $875 \mathrm{MHz}$. This frequency lies in the band below $1 \mathrm{GHz}$ utilized for machine-type communications, [36]. To evaluate the influence of the equalizer error, we considered two extreme cases of channel estimators so as the performance of all practically-feasible estimators will lie in between these two cases. The LMMSE channel estimator with an ideal covariance matrix represents one of the best estimators. As the worst case estimator, we considered a combination of the least squares (LS, for SINR < $14.2 \mathrm{~dB}$ ) and 2D spreading-based (for SINR > 14.2 dB), [32] estimators with MSE curves shown in Fig. 5. We approximated the MSE curves for these two estimators by an exponential regression:

$$
\mathrm{MSE} \approx a \cdot \exp (b \cdot \mathrm{SNR}) .
$$

Fitted MSE curves are shown by dotted and dashed curves in Fig. 5. The exponential regression parameters $a$ and $b$ are shown in Table I. 
TABLE I

PARAMETERS OF EXPONENTIAL REGRESSION FOR THE BEST CASE AND THE WORST CASE ESTIMATORS

\begin{tabular}{|c|c|c|c|}
\hline \multicolumn{2}{|c|}{ Estimator } & $a$ & $b$ \\
\hline Best case & LMMSE ideal CM & 0.624 & -0.219 \\
\hline \multirow{2}{*}{ Worst case } & LS, for SINR < 14.2 dB & 10.376 & -0.287 \\
\cline { 2 - 4 } & 2D spr. est., for SINR > 14.2 dB & 0.168 & 0.003 \\
\hline
\end{tabular}

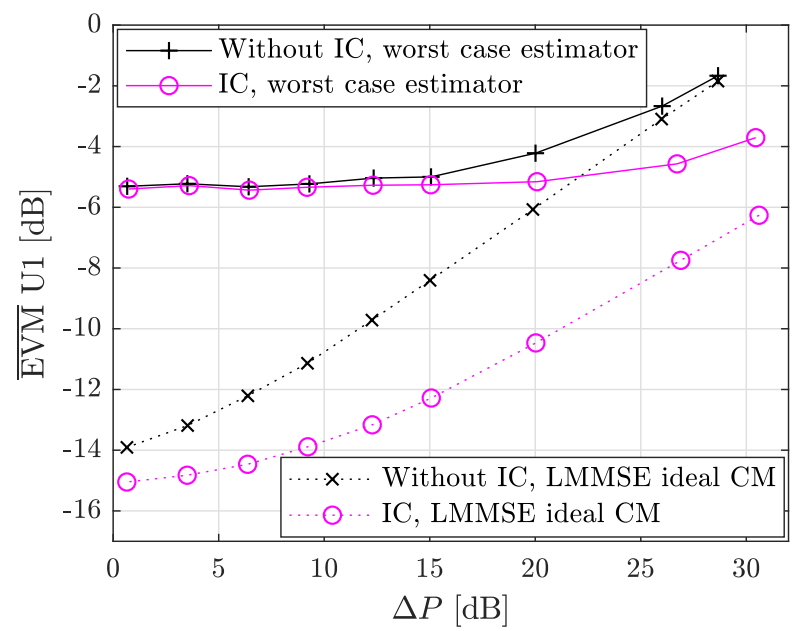

Fig. 8. IC method performance employing frequency filtering, channel estimation error applied to both users (U1+U2) for the best and worst case channel estimators, user $\mathrm{U} 1 \mathrm{SNR}=30 \mathrm{~dB}$.

\section{A. Influence of power difference $\Delta P$}

The performance of the proposed IC method has been evaluated first in terms of EVM, as a function of power difference $\Delta P$ between users. We calculate the EVM value by averaging it over all $L_{\text {data }}=K_{\text {data }} M$ data symbols transmitted on $K_{\text {data }}$ subcarriers:

$$
\overline{\mathrm{EVM}}=10 \cdot \log _{10}\left(\frac{\sum_{l \in \mathcal{L}_{\mathrm{data}}}\left(\hat{d}_{u, l}-d_{u, l}\right)^{2}}{\sum_{l \in \mathcal{L}_{\text {data }}}\left(d_{u, l}\right)^{2}}\right),
$$

where $\mathcal{L}_{\text {data }}$ is a set of all $L_{\text {data }}$ data symbols out of a total of $L$ symbols. The simulation results in terms of averaged EVM of user $U 1$ as a function of power difference $\Delta P[\mathrm{~dB}]$ are shown in Fig. 6-8. Note that for all EVM simulations, the AWGN noise power is $30 \mathrm{~dB}$ below the U1 signal power. To simulate path loss attenuation, the signal of each user was linearly attenuated so that the main channel power of each user is in the range from $-35 \mathrm{dBm}$ to $-3 \mathrm{dBm}$. This allows to control the power difference between the two users.

Fig. 6 shows the IC performance with various filtering methods in the AWGN channel. The black curve describes the case without IC, when U1 EVM is rising with increasing power difference between users U2 and U1. The blue curve describes the IC performance without filtering. For the comparable powers of users U1 and U2, the PA coefficients estimation is strongly affected by the presence of unfiltered U1 and the EVM is degraded severely.

The red and magenta curves correspond to IC employing time domain and frequency domain filtering. In the case of time domain filtering (red curve), the U1 signal is completely filtered out together with the U2 OOB spectral components in its left adjacent band. Note that if filtering is performed on a low intermediate frequency, the spectral components in the right adjacent channel of U2 remain unchanged (see Fig. 4) and contribute to the PA estimation.

The magenta curve corresponds to the case of an IC with frequency domain filtering. The signal U1 together with the U2 spectral components in its left adjacent channel are not filtered out completely, but are shaped by the filter $\mathbf{w}$. Irrespective to the power difference $\Delta P$, interference cancellation with any of the two filtering approaches improves EVM performance over the case with no IC. For equal U1 and U2 power, the IC with filtering restores EVM values close to the ideal EVM of GFDM limited by U1 SNR. For a very weak U1 signal, i.e., $\Delta P>16 \mathrm{~dB}$ for time domain filtering or $\Delta P>27 \mathrm{~dB}$ for frequency domain filtering, filtering of $\mathrm{U} 1$ is not advantageous as removed OOB components of U2 deteriorate U2 power amplifier estimation.

Fig. 7 and Fig. 8 show the IC performance under fading channels with an erroneous channel estimation at the receiver. Fig. 7 shows the IC method performance with considering the estimation error only at the U2 signal demodulator, while 


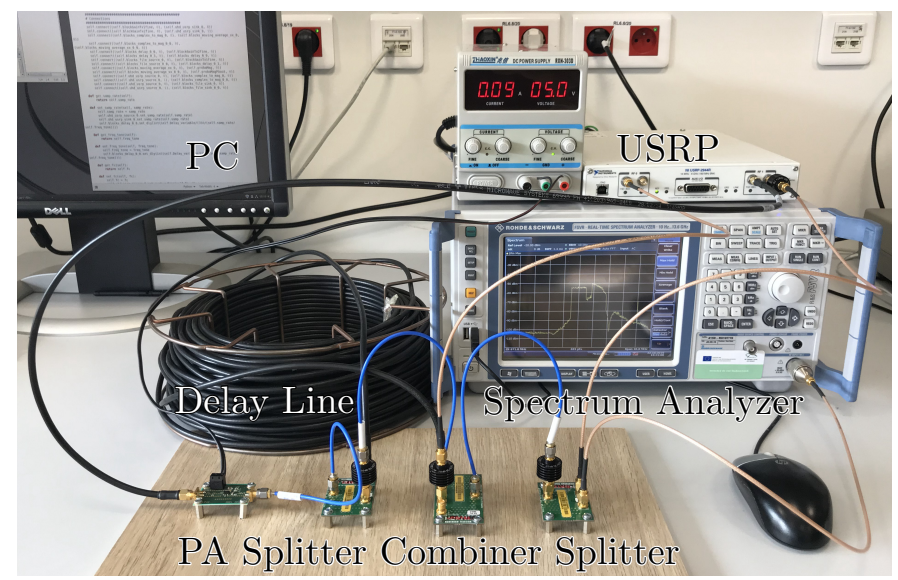

(a) Measurement test bench.

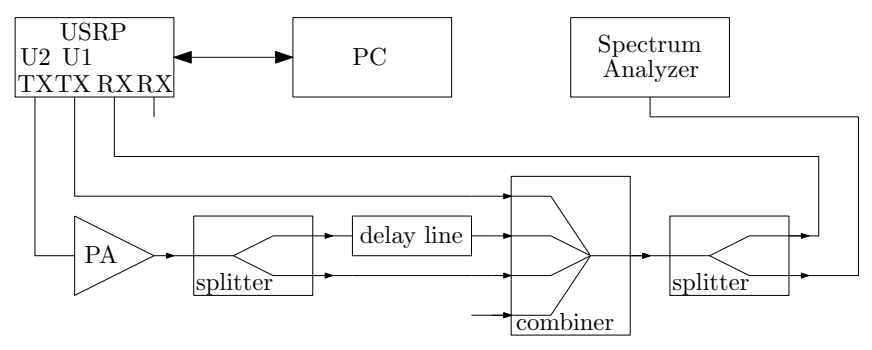

(b) Test bench scheme.

Fig. 9. Measurement setup.

the genie-aided perfect knowledge of U1 CSI is considered. EVM results for the best case channel estimator (LMMSE with ideal $\mathrm{CM}$ ) are depicted by a dotted line and EVM results for the worst case estimator (combination of LS with 2D spreading estimator) are depicted by a solid line.

Fig. 8 depicts the proposed IC method performance with considering channel estimator error for both U1 and U2 user signal demodulation. Here again, EVM of both the best and the worst case estimators is plotted. For the low power difference $\Delta P$ between users, the limiting EVM floor is caused by MSE floor of the 2D spreading estimator at high SINR values.

\section{B. BER performance}

In addition to the EVM performance analysis shown above, the proposed approach was also thoroughly evaluated in terms of BER. For the presentation of BER performance results, we have chosen the fixed power ratio between users $\Delta P=12 \mathrm{~dB}$ and 16-QAM mapping on the subcarriers. The BER curves as a function of SNR are shown in Fig. 10 for the case of the LMMSE estimator with ideal covariance matrix (dotted lines) and for the worst case estimator (solid lines). To approach a slightly more realistic scenario we considered both U2 and U1 signals being amplified with the same nonlinear power amplifier model according to Eq. (8), resulting in an ACPR of approximately $38 \mathrm{~dB}$ in both cases. In addition, we also show the BER results for the case of a nonlinear amplifier only on user $\mathrm{U} 2$ while the signal of user $\mathrm{U} 1$ is amplified linearly by a brown solid line with diamond markers. In the former case, there is a BER floor for high SNR due to the nonlinearity on both PA's, while this is not the case if the PA in U1 is linear.

As a reference state-of-the-art method to compare the performance with, we have selected the ACI approach from [26] originally proposed for single-carrier systems, and we tailored it to the multicarrier GFDM case. In order to make the methods comparable, we did not use any Forward Error Correction (FEC) for both methods and we used the same approach described in Section V for channel-estimator chain modeling. In all cases we considered a perfect synchronization of U1 and U2 signal reception, as in [26]. The most important difference between the proposed and the reference method is that one expects two separate RF front-ends with analog band-selection filters. We have modeled these filters by a 9-th order Butterworth approximation with more than $30 \mathrm{~dB}$ attenuation in the stopband and a maximal attenuation in the passband of $0.5 \mathrm{~dB}$. Note that the requirements on the analog filter have been relaxed due to relatively wide guard band (more than $1 / 3$ of the useful bandwidth) between the users. For a fair comparison, an equivalent filter with bandwidth allowing to capture the signals of both U1 and U2 users and their third order intermodulation products was used in the proposed simulation approach. The results 


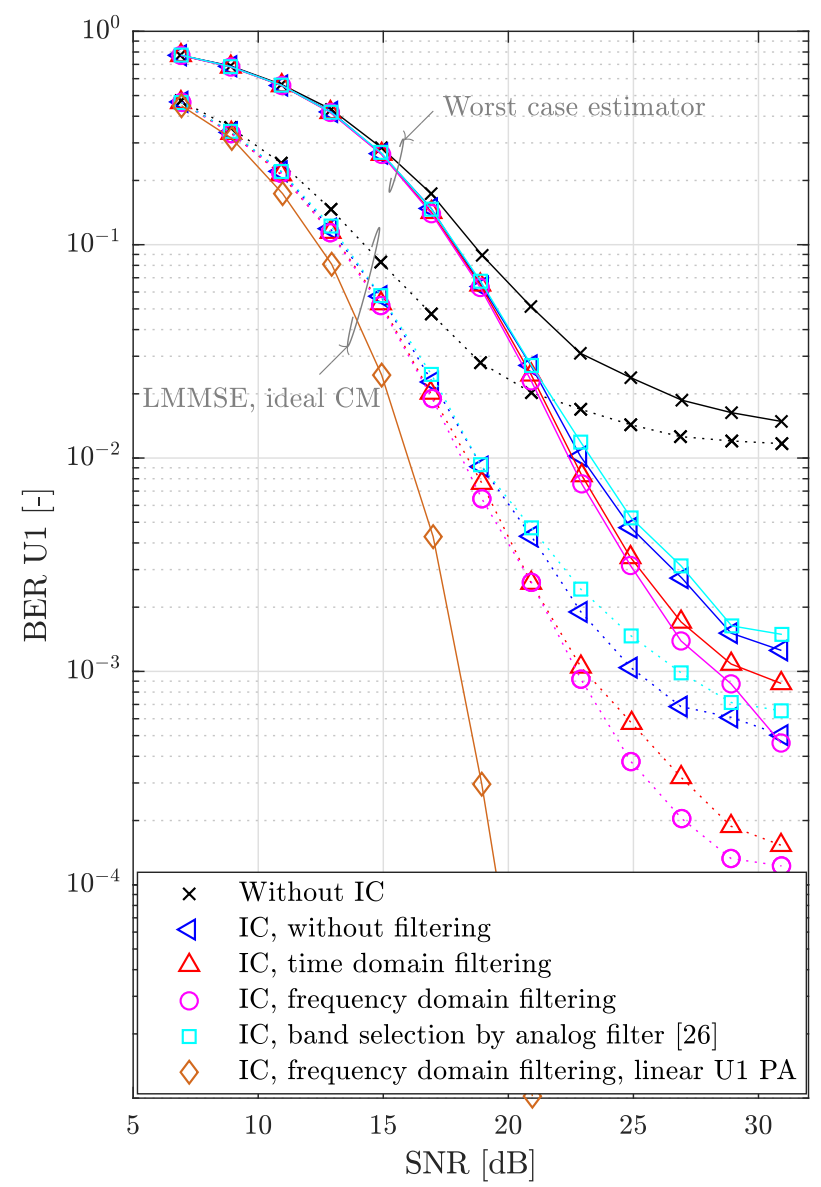

Fig. 10. BER performance for 16-QAM and $\Delta P=12 \mathrm{~dB}$, LMMSE estimator with ideal covariance matrix (solid line), the worst case channel estimator (dotted line)

for the reference method are shown by cyan color in Fig. 10. We can conclude that the proposed approach with separation between users implemented in a digital way outperforms the case of band selection in the analog domain due to the non-ideal filter transfer function. Note that the difference in the performance of methods will depend on the particular realization of the analog filter and on the guard band between the users.

An important question to answer is how the BER improvement due to IC depends on the channel properties, namely on a Root-Mean-Square (RMS) delay spread. We have simulated the proposed IC method in the presence of multipath fading with the RMS delay spread varied from $1.2 \mu s$ corresponding to very mild fading, through $5.9 \mu s$ well covering most of the locations from a measurement campaign [37], to the channel with severe fading with $19 \mu s$ of RMS delay spread. We have considered uncorrelated, Rayleigh-distributed channel taps. At the receiver, a standard Zero Forcing (ZF) equalizer has been used. The channel estimator error of two cases of estimators from Table I has also been modeled. The simulation results are shown on Fig. 11 for the LMMSE estimator with ideal covariance matrix and on Fig. 12 for the worst case channel estimator. A noticeable error floor present without IC for all investigated channel settings is removed with the application of the proposed IC with frequency domain filtering. Although the obtained BER improvement depends on the severity of channel fading, the proposed IC method allows to restore the BER performance very close to the case without ACI, i.e., with linear PA at U2. To illustrate differences in fading character for the three aforementioned channels, we show three example realizations of the channel transfer function magnitudes on Fig. 13.

\section{MEASUREMENT ON THE SDR PlatForm}

In addition to simulations, the proposed method was evaluated by the experiment on the SDR platform based on the NI USRP 2944R with UBX front-end (Fig. 9). The same GFDM waveforms as in the simulations were applied for measurements. The experimental setup consists of the USRP device acting both as the receiver and the transmitter. Coherence between the transmitted and the received signals is obtained by employing the sub-sample time synchronization algorithm [38]. The 


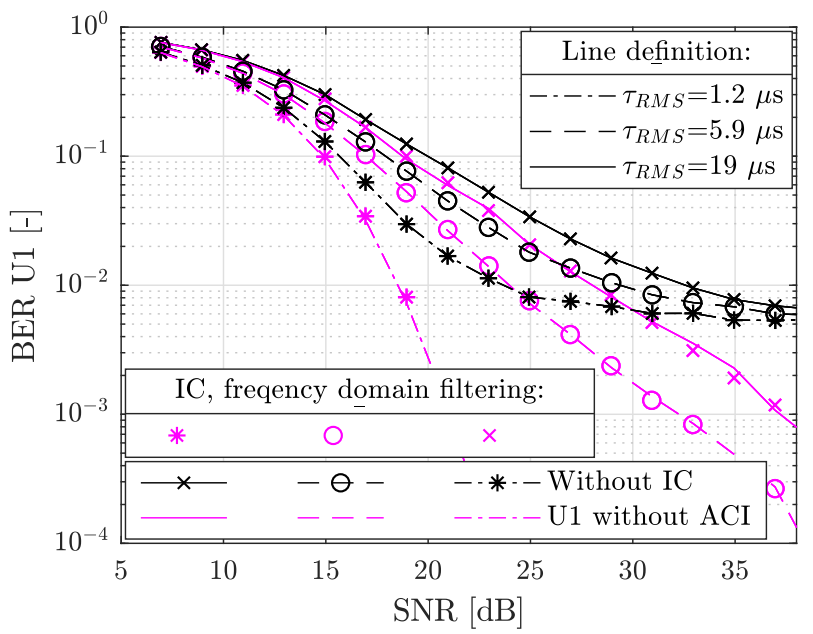

Fig. 11. BER performance without IC (black curves) and with IC (magenta markers) in multipath fading channels with different RMS delay spread $\tau_{R M S}$, ZF equalizer+LMMSE ideal CM channel estimator, 16-QAM and $\Delta P=12 \mathrm{~dB}$. As a reference, BER for U1 without ACI, i.e., with linear PA at U2 (magenta curves) is also plotted.

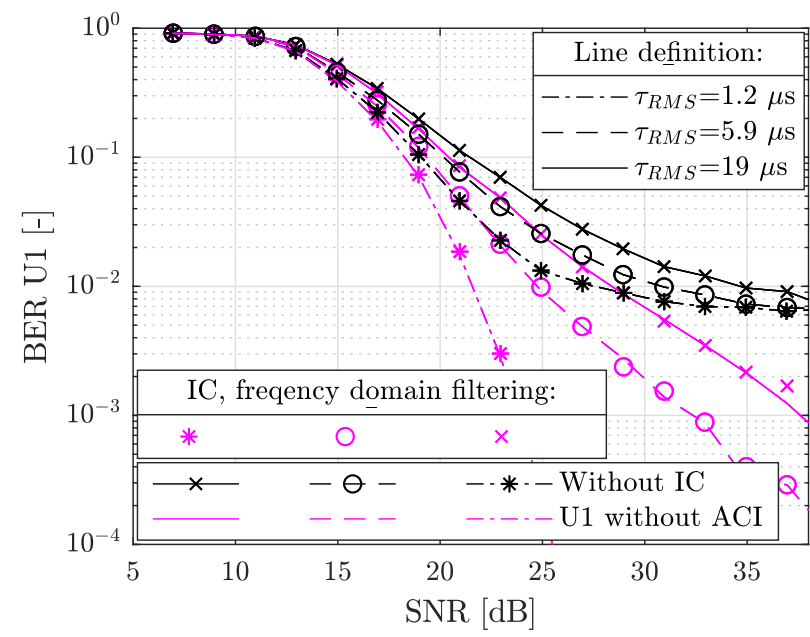

Fig. 12. BER performance without IC (black curves) and with IC (magenta markers) in multipath fading channels with different RMS delay spread $\tau_{R M S}$, ZF equalizer+the worst case channel estimator, 16-QAM and $\Delta P=12 \mathrm{~dB}$. As a reference, BER for U1 without ACI, i.e., with linear PA at U2 (magenta curves) is also plotted.

ADL5610 is used as a power amplifier at the U2 transmitter (externally connected to our USRP device), resulting in the same ACPR of $38 \mathrm{~dB}$ as in the EVM simulations described above. Similarly to EVM simulations, the U1 transmitter has been working in a quasi-linear region with only an internal power amplifier and an ACPR of the signal of approximately $52 \mathrm{~dB}$. Measurements were performed for two scenarios. In the first scenario, the channel between receiver and transmitter was not considered and the transmitters were connected to the receiver through a coaxial cable to verify the functionality of the IC approach in idealized conditions. The second scenario contains a simple channel emulation by a set of splitters and combiners to emulate the signal reflection and attenuation. The U2 signal is split into two paths - the signal in the main path is attenuated by a $20 \mathrm{~dB}$ attenuator and connected with the receiver with sufficiently short cables. The second (reflected) path is created by a $50 \mathrm{~m}$ long RG58 coaxial cable acting as a delay line with a delay of $250 \mathrm{~ns}$ equivalent to 5 samples. The values of the remaining attenuators in this model were chosen to reach U1 SNR at the receiver equal to $30 \mathrm{~dB}$.

Fig. 14 depicts the comparison of simulation results and measurements without channel emulation. Without the IC method applied, there is almost perfect agreement between the simulation and measurement results. With the IC method applied, there is only slight difference between the simulation and measurements. For equal user powers, i.e., $\Delta P$ close to $0 \mathrm{~dB}$, this difference is caused by the residual impairments of the USRP RF frontend, while for high $\Delta P$ values by the simplified PA model used in the simulations.

Results for the case of emulated multipath channel are shown in Fig. 15. The emulated channel was left uncompensated to get significant equalization error. In order to match the simulation conditions with the measurement, the channel was estimated 


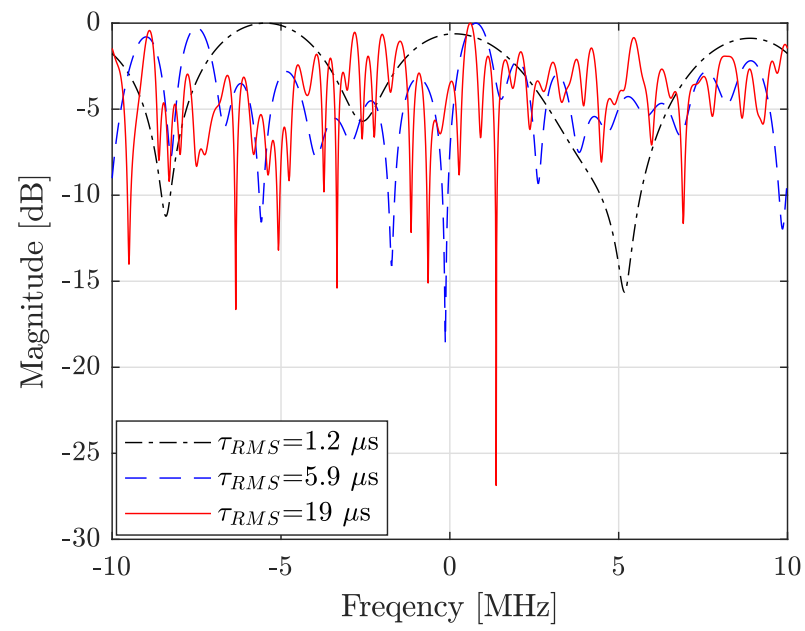

Fig. 13. Examples of used channel transfer function magnitudes for fading channels with three different RMS delay spreads

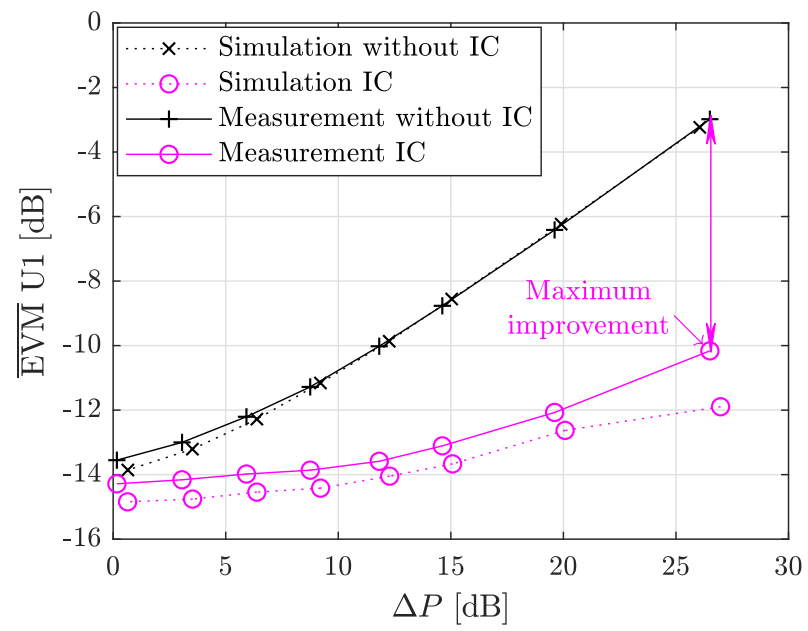

Fig. 14. Comparison of simulation and measurement for the frequency domain filtering IC method without channel emulation, user U1 SNR=30 dB.

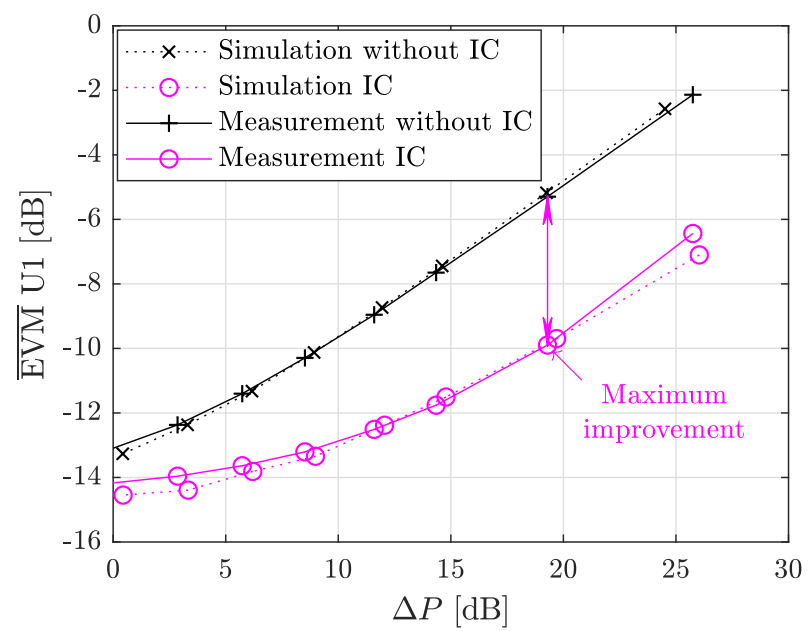

Fig. 15. Comparison of simulation and measurement for the frequency domain filtering IC method with channel emulation, user U1 SNR=30 dB.

using an LS estimator and introduced back in the simulations. Also in this scenario, there is very good agreement between 


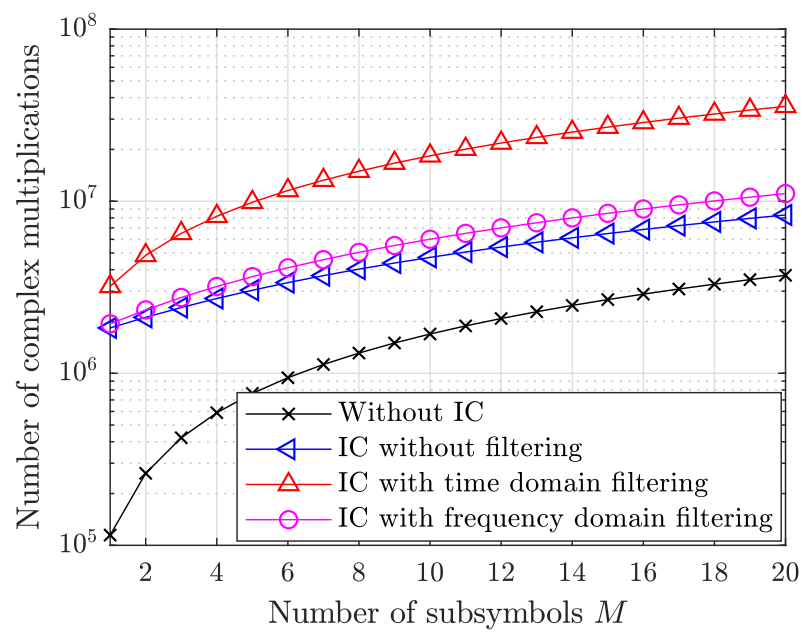

Fig. 16. Comparison of implementation complexity for various $M$.

simulation and measurement results. Further, the measured performance lies well in between the performance of the best case and the worst case estimators from Fig. 8.

The maximum achieved EVM improvement due to the proposed IC method is approximately $7.5 \mathrm{~dB}$ in the case of measurements without a channel and approximately $5 \mathrm{~dB}$ in emulated channel conditions, as shown by magenta arrows in Fig. 14 and Fig. 15. In the example of typical EVM limits as a function of modulation order and FEC rate shown in Table II we want to emphasize that such an EVM improvement allows for the use of higher modulation order (e.g. QPSK instead of BPSK) on the individual subcarriers or higher code rate.

TABLE II

EXAMPLE OF TYPICAL EVM LIMITS FOR AN OFDM-BASED SYSTEM [39]

\begin{tabular}{|c|c|c|}
\hline Inner modulation scheme & Code Rate & EVM Limit (802.11a/g) \\
\hline BPSK & $1 / 2$ & $-5 \mathrm{~dB}$ \\
\hline BPSK & $3 / 4$ & $-8 \mathrm{~dB}$ \\
\hline QPSK & $1 / 2$ & $-10 \mathrm{~dB}$ \\
\hline QPSK & $3 / 4$ & $-13 \mathrm{~dB}$ \\
\hline 16-QAM & $1 / 2$ & $-16 \mathrm{~dB}$ \\
\hline 16-QAM & $3 / 4$ & $-19 \mathrm{~dB}$ \\
\hline 64-QAM & $2 / 3$ & $-22 \mathrm{~dB}$ \\
\hline 64-QAM & $3 / 4$ & $-25 \mathrm{~dB}$ \\
\hline
\end{tabular}

\section{COMPLEXITY OF THE METHOD}

The price to pay for the demonstrated EVM and BER improvement is the increased complexity of the receiver employing the IC. Our interference scenario as defined in Fig. 1, in general, may consider not only uplink, but also downlink. The complexity will be discussed for the most common uplink scenario as in [26] or [27] only. In such a case there is no additional hardware complexity related to our method as the receiver needs to process both U1 and U2 signals anyway.

In the uplink receiver, both signals need to be received, frequency shifted and demodulated. Thus, Step 1 and 5 from the algorithm as described in Section III-A do not contribute to a complexity increase. The complexity of the remaining blocks is summarized in Table III in terms of a number of complex multiplications. For the GFDM modulator/demodulator, we consider the optimized FFT-based implementation according to [40] and we assume that a $D$-point discrete transform can be implemented with the FFT algorithm at the expense of $D \log _{2} D$ complex multiplications. The standard GFDM receiver without IC needs just one GFDM demodulator block for each of the U1 and U2 signals. Any of the expected IC methods requires an additional GFDM modulator block for interfering user data re-modulation (Step 2 in Section III-A), the nonlinear PA estimation block (Step 3 in Section III-A) and the PA model application block (inside Step 4 in Section III-A). The implementation of corresponding filters contribute to the overall complexity of the IC methods with time or frequency domain filtering. Note that for time domain filtering (Case 1 in Section III-B) we consider a direct-form FIR filter. We assume the least squares estimation of nonlinear PA characteristics on one subsymbol of $K=4096$ samples, as such length was demonstrated to be sufficient for simple low-order PA models in several papers, including [41]. 
Fig. 16 shows the total number of complex multiplications for considered methods as a function of the number of GFDM subsymbols $M$. Together with simulation and measurement results, it shows that the IC method with the frequency domain filtering results in the best EVM and BER, with only slightly increased complexity compared to the method without a digital filter. Note that equivalent operations need to be performed also in the case of other state-of-the-art methods, e.g. [26], except the frequency-domain filtering between users.

TABLE III

COMPLEXITY OF INDIVIDUAL BLOCKS OF GFDM RECEIVER WITH IC

\begin{tabular}{|c|c|}
\hline Operation & Complexity \\
\hline GFDM mod./demod. [40] & $M K \log _{2}(K)+2 K M \log _{2}(M)+2 K M$ \\
\hline PA estimation [41], [42] & $2 K(P(Q-1))^{2}$ \\
\hline PA application & $P(Q-1)-1$ \\
\hline Time domain filtering & $b_{F I R} K M$ \\
\hline Freq. domain filtering & $2 K M \log _{2}(K M)+K M$ \\
\hline
\end{tabular}

$b_{F I R}=333$ is number of coefficients of the FIR filter

\section{COnclusions}

The interference between collocated users transmitting with unequal power on nearby frequency slots is nowadays an important problem in vehicular or other machine-type communications. In this paper we have presented a new interference cancellation approach to suppress adjacent channel interference caused by a nonlinear power amplifier. The over-the-air estimation of a nonlinear power amplifier model is an important part of the described method. We thus proposed digital frequency domain filtering of adjacent signals to obtain a re-generated replica of the transmitted signal, necessary for the least squares adaptation of the power amplifier model.

We have demonstrated the method and its performance on the example of GFDM signals. The method has been evaluated in the scenario of equal user powers, but also in the case when the powers of users differ significantly. Besides simulations, the proposed approach has also been tested on the SDR-based measurement setup, with sufficiently good agreement between measurement and simulation results.

Without considering channel estimation errors, the maximal EVM improvement due to the proposed method in the given configuration is around $7 \mathrm{~dB}$. In the range of power difference between users from 10 to $25 \mathrm{~dB}$, the application of the proposed method leads to an EVM improvement of approximately $5 \mathrm{~dB}$ allowing for increasing modulation order on individual subcarriers or for using higher FEC code rates. Only for a power difference between users below $10 \mathrm{~dB}$, the EVM improvement decreases to $2 \mathrm{~dB}$, but in this region the demodulation performance is already good and limited by the receiver SNR and by the residual impairments of the RF front-end.

Further, we have shown that a robust channel estimation is a key aspect for the successful implementation of the proposed method. The LMMSE estimator leads to EVM improvement worse by approximately $1 \mathrm{~dB}$ compared to the case of perfect CSI and even for practical channel estimators, the proposed method still has a potential to provide signal quality improvement. This has also been verified by the simulations on a model of a frequency selective channel with RMS delay spread ranging from $1.2 \mu \mathrm{s}$ to $19 \mu \mathrm{s}$. By extensive BER simulations, we have shown that the proposed method has the potential to overcome the drawback of similar state-of-the-art approaches, that is the band selection by realistic, and thus non-ideal, analog bandpass filters. The complexity analysis shows that the proposed IC method with the frequency domain filtering has only insignificantly higher computational demands, but can bring considerable improvement of the BER/EVM performance. 


\section{REFERENCES}

[1] M. Bellanger, "Physical Layer for Future Broadband Radio Systems," in IEEE Radio and Wireless Symposium (RWS), Jan 2010, pp. 436-439.

[2] V. Vakilian, T. Wild, F. Schaich, S. ten Brink, and J. Frigon, "Universal-Filtered Multi-Carrier Technique for Wireless Systems Beyond LTE," in IEEE Globecom Workshops (GC Wkshps), Dec 2013, pp. 223-228.

[3] J. Abdoli, M. Jia, and J. Ma, "Filtered OFDM: A New Waveform for Future Wireless Systems," in IEEE International Workshop on Signal Processing Advances in Wireless Communications (SPAWC), June 2015, pp. 66-70.

[4] W. Cao, L. Zhang, W. Hu, J. Lei, and X. Du, "OFDM Ambiguity Function Improvement with FBMC Prototype Filter for Passive Radar," in International Radar Symposium (IRS), June 2017, pp. 1-9.

[5] N. Michailow, M. Matthé, I. S. Gaspar, A. N. Caldevilla, L. L. Mendes, A. Festag, and G. Fettweis, "Generalized Frequency Division Multiplexing for 5th Generation Cellular Networks," IEEE Transactions on Communications, vol. 62, no. 9, pp. 3045-3061, Sept 2014.

[6] T. Gotthans, R. Maršálek, J. Blumenstein, and G. Baudoin, "Experimental Evaluation of Digital Predistortion with FBMC and OFDM Signals," in IEEE Annual Wireless and Microwave Technology Conference (WAMICON 2015), April 2015, pp. 1-3.

[7] D. Dardari, V. Tralli, and A. Vaccari, "A Theoretical Characterization of Nonlinear Distortion Effects in OFDM Systems," IEEE Transactions on Communications, vol. 48, no. 10, pp. 1755-1764, Oct 2000.

[8] J.Li and M. Kavehrad, "OFDM-CDMA systems with nonlinear power amplifier," in WCNC. IEEE Wireless Communications and Networking Conference (Cat. No.99TH8466), vol. 3, Sep. 1999, pp. 1167-1171 vol.3.

[9] T. Lee and H. Ochiai, "A simple characterization of power spectral density for nonlinearly amplified OFDM signals," in IEEE Topical Conference on Power Amplifiers for Wireless and Radio Applications (PAWR), Jan 2014, pp. 88-90.

[10] R. Maršálek, P. Jardin, and G. Baudoin, "From Post-Distortion to Pre-Distortion for Power Amplifiers Linearization," IEEE Communications Letters, vol. 7, no. 7, pp. 308-310, July 2003.

[11] S. V. Zhidkov, "Receiver Synthesis for Nonlinearly Amplified OFDM Signal," in IEEE Workshop on Signal Processing Systems (IEEE Cat. No.03TH8682), Aug 2003, pp. 387-392.

[12] J. Tellado, L. M. C. Hoo, and J. M. Cioffi, "Maximum-Likelihood Detection of Nonlinearly Distorted Multicarrier Symbols by Iterative Decoding," IEEE Transactions on Communications, vol. 51, no. 2, pp. 218-228, Feb 2003.

[13] L. Sendrei and S. Marchevský, "Nonlinear Noise Estimation and Compensation in GFDM Based Communication Systems for Cognitive Radio Networks," in International Conference Radioelektronika (RADIOELEKTRONIKA), April 2015, pp. 313-316.

[14] H. Bouhadda, R. Zayani, H. Shaiek, D. Roviras, and R. Bouallegue, "Iterative Receiver Cancellation of Nonlinear Power Amplifier Distortion in FBMC/OQAM System," in IEEE International Conference on Wireless and Mobile Computing, Networking and Communications (WiMob), Oct 2015, pp. 691-695.

[15] R. Nissel, M. Rupp, and R. Maršálek, "FBMC-OQAM in Doubly-Selective Channels: A New Perspective on MMSE Equalization," in IEEE International Workshop on Signal Processing Advances in Wireless Communications (SPAWC), July 2017, pp. 1-5.

[16] R. Datta, N. Michailow, M. Lentmaier, and G. Fettweis, "GFDM Interference Cancellation for Flexible Cognitive Radio PHY Design," in IEEE Vehicular Technology Conference (VTC Fall), Sept 2012, pp. 1-5.

[17] J. Zhong, G. Chen, J. Mao, S. Dang, and P. Xiao, "Iterative Frequency Domain Equalization for MIMO-GFDM Systems," IEEE Access, vol. 6, pp. 19386-19395, 2018

[18] L. Rudant, R. D’Errico, M. Robin, J. Zeleny, P. Rosson, C. Dehos, and A. Kaiser, "Over-The-Air Validation of PA Nonlinearity Estimation At the Receiver," in European Conference on Antennas and Propagation (EuCAP), April 2013, pp. 308-311.

[19] Q. Luo, C. Yu, and X. Zhu, "Digital Predistortion of Phased Array Transmitters with Multi-Channel Time Delay," in IEEE Topical Conference on RF/Microwave Power Amplifiers for Radio and Wireless Applications (PAWR), Jan 2018, pp. 54-57.

[20] Z. L. et al. (2018) Intermediate 5G V2X radio, version 1.0. [Online]. Available: https://5gcar.eu/wp-content/uploads/2018/08/5GCAR_D3.1_v1.0.pdf

[21] S. Wang, J. Armstrong, and J. S. Thompson, "Waveform Performance for Asynchronous Wireless 5G Uplink Communications," in IEEE Annual International Symposium on Personal, Indoor, and Mobile Radio Communications (PIMRC), Sep. 2016, pp. 1-6.

[22] G. Zhao, "Method, modem, and system for canceling adjacent channel interference," Patent US9008 250B2, April 14, 2015.

[23] M. Lopez, "Adjacent channel interference supression," Patent US20 070254 590A1, February 26, 2006.

[24] R. Morelos-Zaragoza and S. Kuruba, "An adaptive adjacent channel interference cancellation technique," in 2009 Global Mobile Congress, Oct 2009, pp. 1-4.

[25] M. T. Nguyen and L. B. Le, "Adjacent channel interference cancellation for robust spectrum sharing in satellite communications systems," in 2017 IEEE 28th Annual International Symposium on Personal, Indoor, and Mobile Radio Communications (PIMRC), Oct 2017, pp. 1-5.

[26] N. Osawa, S. Ibi, and S. Sampei, "Iterative canceller of adjacent channel interference induced by nonlinearity of power amplifier in millimeter wave systems," in 2015 IEEE 26th Annual International Symposium on Personal, Indoor, and Mobile Radio Communications (PIMRC), Aug 2015, pp. 781-786.

[27] H. C. Nguyen, E. de Carvalho, and R. Prasad, "Multi-user interference cancellation schemes for carrier frequency offset compensation in uplink OFDMA," IEEE Transactions on Wireless Communications, vol. 13, no. 3, pp. 1164-1171, March 2014.

[28] L. Lecheminoux and M. Villegas, "ACPR to third order interception point and $1 \mathrm{~dB}$ compression point new relationship," in 2000 30th European Microwave Conference, Oct 2000, pp. 1-4.

[29] J. Kim and K. Konstantinou, "Digital Predistortion of Wideband Signals Based on Power Amplifier Model with Memory," Electronics Letters, vol. 37, no. 23, pp. 1417-1418, Nov 2001.

[30] E. Ozturk, E. Basar, and H. A. Çirpan, "Generalized Frequency Division Multiplexing With Flexible Index Modulation Numerology," IEEE Signal Processing Letters, vol. 25, no. 10, pp. 1480-1484, Oct 2018.

[31] Y. Liu, W. Pan, S. Shao, and Y. Tang, "A General Digital Predistortion Architecture Using Constrained Feedback Bandwidth for Wideband Power Amplifiers," IEEE Transactions on Microwave Theory and Techniques, vol. 63, no. 5, pp. 1544-1555, May 2015.

[32] J. Blumenstein, R. Maršálek, Z. Fedra, A. Prokeš, and C. Mecklenbräuker, "Channel Estimation Method for OFDM in Low SNR Based on TwoDimensional Spreading," Wireless Personal Communications, vol. 78, no. 1, pp. 715-728, 2014.

[33] C. Wang, E. K. S. Au, R. D. Murch, W. H. Mow, R. S. Cheng, and V. Lau, "On the Performance of the MIMO Zero-Forcing Receiver in the Presence of Channel Estimation Error," IEEE Transactions on Wireless Communications, vol. 6, no. 3, pp. 805-810, March 2007.

[34] V. C. M. C. S. Technical University Dresden. (2014) GFDM Downloads TU Dresden. [Online]. Available: http://owl.ifn.et.tu-dresden.de/GFDM/

[35] Keysight Technologies Inc., "LTE-Advanced Physical Layer Overview." [Online]. Available: http://rfmw.em.keysight.com/wireless/helpfiles/89600b/ webhelp/subsystems/lte-a/content/lte_overview.htm

[36] Nokia, "5G for people and things, $700 \mathrm{MHz}$ band as key success for wide-area 5G services," accessed: 2019-02-24. [Online]. Available: https://www.itu.int/en/ITU-D/Regional-Presence/Europe/Documents/Events/2017/Spectrum\%20Management/Ulrich_Nokia_5G_in\%20700w.pdf

[37] S. Y. Seidel, T. S. Rappaport, and R. Singh, "Path loss and multipath delay statistics in four european cities for $900 \mathrm{MHz}$ cellular and microcellular communications," Electronics Letters, vol. 26, no. 20, pp. 1713-1715, 1990.

[38] J. Kral, T. Gotthans, and M. Harvanek, "Analytical method of fractional sample period synchronisation for digital predistortion systems," in International Conference Radioelektronika (RADIOELEKTRONIKA), April 2017, pp. 1-5.

[39] National Instruments, "Introduction to WLAN Testing." [Online]. Available: http://download.ni.com/evaluation/rf/Introduction_to_WLAN_Testing.pdf

[40] N. Michailow, I. Gaspar, S. Krone, M. Lentmaier, and G. Fettweis, "Generalized frequency division multiplexing: Analysis of an alternative multi-carrier technique for next generation cellular systems," in 2012 International Symposium on Wireless Communication Systems (ISWCS), Aug 2012, pp. 171-175. 
[41] P. M. Suryasarman and A. Springer, "A comparative analysis of adaptive digital predistortion algorithms for multiple antenna transmitters," IEEE Transactions on Circuits and Systems I: Regular Papers, vol. 62, no. 5, pp. 1412-1420, May 2015.

[42] S. Wang, "Study on complexity reduction of digital predistortion for power amplifier linearization," Ph.D. dissertation, 2018.

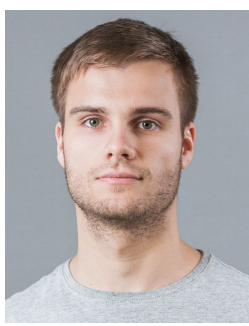

Michal Harvanek received his Bachelor's degree and his Ing. degree (M.Sc. equivalent) in electrical engineering from Brno University of Technology, Brno, Czech Republic, in 2013 and 2015. He has collaborated with CISC Semiconductor in the area of RFID signal processing implementations. He is currently pursuing his Ph.D. degree at the Department of Radio Electronics, Brno University of Technology, Brno, Czech Republic. His research interests include FDM modulation techniques, transceiver impairments, and interference cancellation methods.

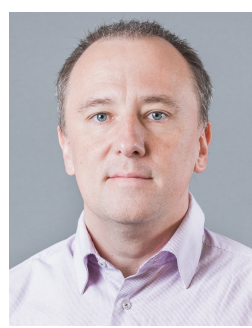

Roman Marsalek received his Ing. degree from Brno University of Technology, Brno, Czech Republic, in 1999 and his Ph.D. degree from Université de Marne-La-Vallée, France, in 2003. In 2013, he was a teaching and research fellow at Johannes Kepler University, Linz, Austria. He is currently a Full Professor at the Department of Radio Electronics, Brno University of Technology. His research interests include wireless communications theory and applied digital signal processing.

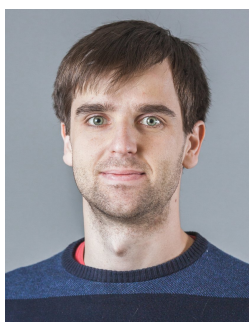

Jan Kral received his Bachelor's degree and his Ing. degree (M.Sc. equivalent) in electrical engineering from Brno University of Technology, Brno, Czech Republic, in 2012 and 2014. From 2013 to 2014, he worked on his diploma thesis, Fast Beam Current Change Monitor for LHC, at CERN, Geneva, Switzerland. He is currently working towards his Ph.D. degree at Brno University of Technology. His research interests include digital predistortion of power amplifiers, transceiver impairments, and application of digital signal processing to FPGAs.

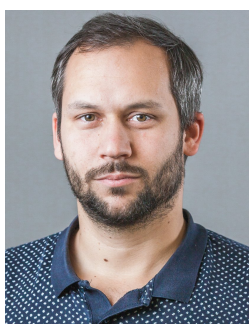

Tomas Gotthans received his Bachelor's degree and his Ing. degree in electrical engineering from Brno University of Technology in 2008 and 2010. In 2011 he joined ESIEE Paris, ESYCOM laboratory where he worked on the project AMBRUN (in collaboration with Thales Communications \& Security, TeamCast, Supélec). In January 2014 he received his Ph.D. degree from the Université de MarneLa-Vallée, France. He is currently an Associate Professor at the Department of Radio Electronics, Brno University of Technology. His research interests include digital predistortion of power amplifiers, wireless communications theory, and chaos theory.

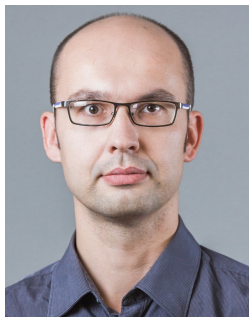

Jiri Blumenstein received his Ph.D. degree from the Brno University of Technology, in 2013. In 2011, he was a Researcher with the Institute of Telecommunications, TU Wien. He has cooperated with several companies, including Racom, Volkswagen, and ON Semiconductor in the area of applied research of wireless systems and in the area of the fundamental research funded by the Czech Science Foundation. He is currently a Researcher with the Department of Radio Electronics, Brno University of Technology. His research interests include signal processing, physical layer of communication systems, channel characterization and modeling, and wireless system design. 


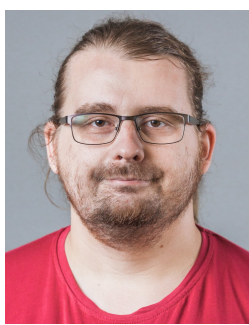

Martin Pospisil was born in Valtice, Czech Republic in 1988. He received his Bachelor degree and his Ing. degree in Electronics and Communication technologies from Brno University of Technology, in 2010 and 2012, respectively. He has cooperated with CISC semiconductor in the area of signal processing implementations for RFID. He is specialized in RF transceiver design and instrumentation and is currently pursuing his Ph.D. degree with a main focus on transmitter identification via RF impairments.

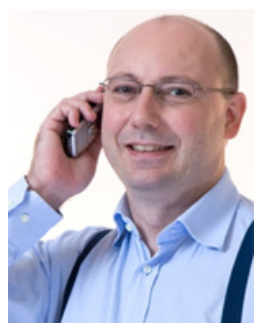

Markus Rupp received his Dipl.-Ing. degree from the University of Saarbrücken, Saarbrücken, Germany, in 1988 and his Dr.-Ing. degree from Technische Universität Darmstadt, Darmstadt, Germany, in 1993. Until 1995, he had a Postdoctoral position with the University of Santa Barbara, CA, USA. From 1995 to 2001, he was with the Wireless Technology Research Department of BellLabs, NJ. Since October 2001, he has been a Full Professor for Digital Signal Processing in Mobile Communications with TU Wien, Vienna, Austria. 